\title{
VELOCIDADE DE HEMOSSEDIMENTAÇÃO (VHS) SEM DILUIÇÃO: METODOLOGIA CONFIÁVEL?
}

\section{UNDILUTED ERYTHROCYTE SEDIMENTATION RATE (ESR): A TRUST FULL METHODOLOGY?}

\begin{abstract}
Rabih Hussein HACHEM ${ }^{1}$, Railson HENNEBERG ${ }^{2}$, Aguinaldo José do
\end{abstract} NASCIMENTO ${ }^{3}$, Rogério Luiz KOPP ${ }^{2}$; Paulo Henrique da SILVA²

\author{
'Aluno do Curso de Especialização em Análises Clínicas da Universidade Federal do Paraná - UFPR. \\ Avenida Lothário Meissner, 632, campus Botânico. Universidade Federal do Paraná. \\ e-mail: rabihhusseinhachem@hotmail.com \\ ${ }^{2}$ Docentes do Curso de Farmácia da Universidade Federal do Paraná - UFPR. \\ e-mails: railson@ufpr.br, paulo.hs@ufpr.br, rlkopp@ufpr.br \\ ${ }^{3}$ Prof. Sênior do Programa de Pós-graduação em Ciências Farmacêuticas da UFPR \\ e-mail: ajnasc@gmail.com REC: 05/2010AC: 06/2010
}

\section{RESUMO:}

Um dos marcadores da resposta inflamatória é a velocidade de hemossedimentação (VHS). Considerado como um dos marcadores mais antigos utilizado na rotina laboratorial como indicador de doença ativa, este teste, sofreu algumas adaptações na sua metodologia em relação ao método de referência (método de Westergren), sendo utilizado sangue total sem diluição. O objetivo deste estudo foi demonstrar se há diferença entre o método de referência e o método alternativo (não-diluído). Os resultados deste estudo demonstraram que na média geral, a não-diluição do sangue com EDTA (método alternativo) não demonstrou nenhuma diferença significativa com relação ao método preconizado. Estratificando os valores de VHS acima de $30 \mathrm{~mm}$, observamos um aumento significativo dos valores pelo método alternativo, ou seja, os valores da VHS por este método tendem a ser maiores do que os obtidos pela técnica com citrato. Os resultados obtidos demonstraram que as adaptações, como a nãodiluição do sangue, podem influenciar a sensibilidade do teste do VHS, e este método alternativo não deve ser usado na rotina laboratorial.

Palavras-chave: velocidade de hemossedimentação, método de Westergren, método de Westergreen alternativo.

\section{ABSTRACT:}

Erythrocyte sedimentation rate (ESR) is a marker of inflammatory disease. It is considered one of the oldest tests used in clinical laboratory as an indicator of active disease. The Westergren method is recommended by International Committee for 
Standardization in Haematology (ICSH), but a technical variation has been introduced with undiluted blood. The purpose of this work was to find out if there is a significative statistical difference between both methods. The average values of ESR of undiluted blood had no statistical difference compared with the ones of reference method. When the results were separated in two classes, lower and higher than $30 \mathrm{~mm}$ a statistical difference was observed: the ESR from undiluted blood is higher than the reference method. The results showed that adaptations from reference method, as undiluted blood, have an influence in ESR sensitivity and the undiluted method should not be used in clinical laboratory.

Key-words: Erythrocyte sedimentation rate, Westergren method

\section{INTRODUÇÃO}

A velocidade de hemossedimentação (VHS) é um teste simples e de baixo custo que tem sido usado, há mais de meio século, como um marcador da resposta inflamatória (COLLARES, 2004). O exame consiste na medida da altura da camada dos eritrócitos, de uma amostra de sangue venoso anticoagulado, que sedimenta em um tudo de vidro graduado num determinado período de tempo. Ahemossedimentação possui três fases: a primeira fase ou de sedimentação inicial, consiste na queda individual dos eritrócitos, antes da sedimentação. A segunda fase, ou de sedimentação máxima, consiste na formação dos agregados globulares, os quais tanto mais depressa sedimentarão quanto maiores e mais numerosos. Na terceira fase, ou de sedimentação constante, os agregados globulares atingem, por unidade de tempo, período de queda constante, a qual entra em declínio no período final, à medida que os glóbulos vão se acumulando na parte inferior da pipeta. Quando os agregados caem, há deslocamento do plasma, que, consequentemente, forma uma corrente para cima, retardando a queda dos agregados (DICKINSON, 1998).

Vários fatores podem afetar o resultado da VHS produzindo resultados pouco confiáveis. Inúmeras variáveis intrínsecas ou extrínsecas contribuem para a baixa especificidade do teste. Apesar destas limitações, ainda hoje, a VHS vem sendo utilizada com frequência na prática clinica como marcador inespecífico de doenças inflamatórias (DICKINSON, 1998).

Entre os fatores que podem alterar de alguma forma os valores da VHS destacam-se o período de jejum, a concentração de fibrinogênio e das globulinas do plasma, a viscosidade do plasma, a concentração do colesterol, a concentração de íons hidrogênio, concentração, tamanho e forma dos eritrócitos e o conteúdo hemoglobínico (DICKINSON,1998). Além disto, a idade e o sexo do paciente devem ser levados em consideração na avaliação do resultado da VHS. Por outro lado, os fatores técnicos também devem ser analisados, pois as condições da coleta do 
sangue, a ocorrênciaou não de estase venosa, a qualidade da homogeneização do sangue interferem diretamente nos resultados do teste (LANZARA,2001).

O teste da VHS como conhecemos hoje, foi introduzido na Alemanha, em 1918, por Robin Fahreus (COLLARES, 2004), que definiu quase todas as suas características importantes, como a relação entre a sedimentação e a capacidade do plasma em reduzir a carga eletrostática na superfície dos eritrócitos, produzindo maior sedimentação. Além disso, ele quantificou a capacidade de sedimentação das proteínas plasmáticas, avaliou o efeito da temperatura na VHS e verificou o aumento da VHS em várias condições patológicas e fisiológicas, entre elas a gestação.

Em 1920, foi feita uma padronização do teste por Westergren, considerada padrão ouro para a VHS, que foi recomendada pelo International Committee for Standardization in Haematology (ICSH) em 1977 (BEDELL,1985, SOX,1986). O método consiste em colocar sangue venoso anticoagulado com citrato de sódio 0,106M (relação 4:1) em um tudo de vidro graduado, com $200 \mathrm{~mm}$ de comprimento e $2,5 \mathrm{~mm}$ de diâmetro interno. O tubo é preenchido até a marca zero e deixado na posição vertical por uma hora. A VHS é expressa em $\mathrm{mm} / \mathrm{h}$, que é a distância do menisco até o topo da coluna de eritrócitos. O exame deve ser feito até duas horas após a coleta e a uma temperatura entre 20 e 25C. Existem algumas variações descritas deste método, como o uso de sangue anticoagulado com EDTA (COLLARES, 2004).

O sangue é coletado com EDTA e posteriormente diluído com citrato de sódio ou salina na proporção de 4:1. Esta modificação apresenta boa relação com o método padrão e passou a ser considerada também como um método de referência para a VHS pela ICSH a partir de 1993. Além disso, ela permite que a mesma amostra de sangue possa ser utilizada para a realização da VHS e outros testes hematológicos e que o exame possa ser realizado até 12 horas após a coleta. Muitos laboratórios realizam a VHS sem fazer a diluição prévia com citrato ou salina, utilizando apenas o sangue coletado com EDTA, método que não é preconizado pela ICSH.

Este trabalho tem como objetivo comparar os métodos de Westergren (padrão ouro) com citrato de sódio a $0,106 \mathrm{M}$ (relação 4:1), com o método alternativo que utiliza apenas o EDTA, sem prévia diluição, para avaliar a fidedignidade entre os dois métodos.

\section{MATERIAL E MÉTODOS}

Foram avaliados os métodos de Westergren (padrão ouro) e o método alternativo, sem diluição. O método de Westergren consiste na diluição de 4:1 de sangue venoso anticoagulado com citrato de sódio a $0,106 \mathrm{M}$, é recomendado pelo International Committee for Standardization in Haematology (ICSH), a partir de 1977, e é considerado como padrão ouro. No método alternativo foi transferido um volume de 


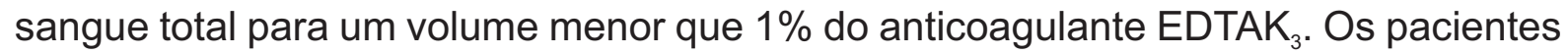
$(n=326)$ foram escolhidos aleatoriamente, sem levar em consideração idade, sexo, diagnóstico clínico e o volume globular. Essa avaliação foi realizada no laboratório UNILAB de Ponta Grossa no período de dezembro de 2008 a maio de 2009.

As amostras foram homogeneizadas, com velocidade constante e em baixa rotação, durante 20 minutos antes de serem pipetadas, sendo retiradas somente no momento da pipetagem.

As duas amostras, de cada paciente, foram pipetadas em uma coluna de vidro de $300 \mathrm{~mm}$ de altura por $2,5 \mathrm{~mm}$ de diâmetro com graduação de $200 \mathrm{~mm}$ na extremidade inferior e $0 \mathrm{~mm}$ na extremidade superior. A VHS foi realizada em temperatura ambiente entre 20 a $25^{\circ} \mathrm{C}$ e ao abrigo da luz solar.

As leituras das VHS foram realizadas após uma hora a partir da coluna de plasma, no limite de separação com as hemácias sedimentadas e o resultado foi expresso em $\mathrm{mm} / \mathrm{h}$.

\section{RESULTADOS}

A tabela 1 mostra as médias e os desvios padrão obtida para cada método.

TABELA 1 - Médias e desvios padrão dos dois métodos de determinação de VHS.

\begin{tabular}{|c|c|c|c|}
\hline VHS & $\mathrm{N}$ & Média & Teste $\mathrm{t}$ \\
\hline sem diluição & 326 & $\begin{array}{c}14,65 \pm \\
18,77\end{array}$ & to $=0,401 ; p=0,689(\mathrm{NS})$ \\
\hline com diluição* & 326 & $14,09 \pm$ & \\
\hline
\end{tabular}

A Figura 1ilustra a análise de correlação entre os dois métodos, demonstrando uma correlação estatisticamente significante $\left(r^{2}=0,959\right)$, com um erro sistemático de $7,4 \%$. 
FIGURA 1. Correlação entre os dois métodos de determinação do VHS

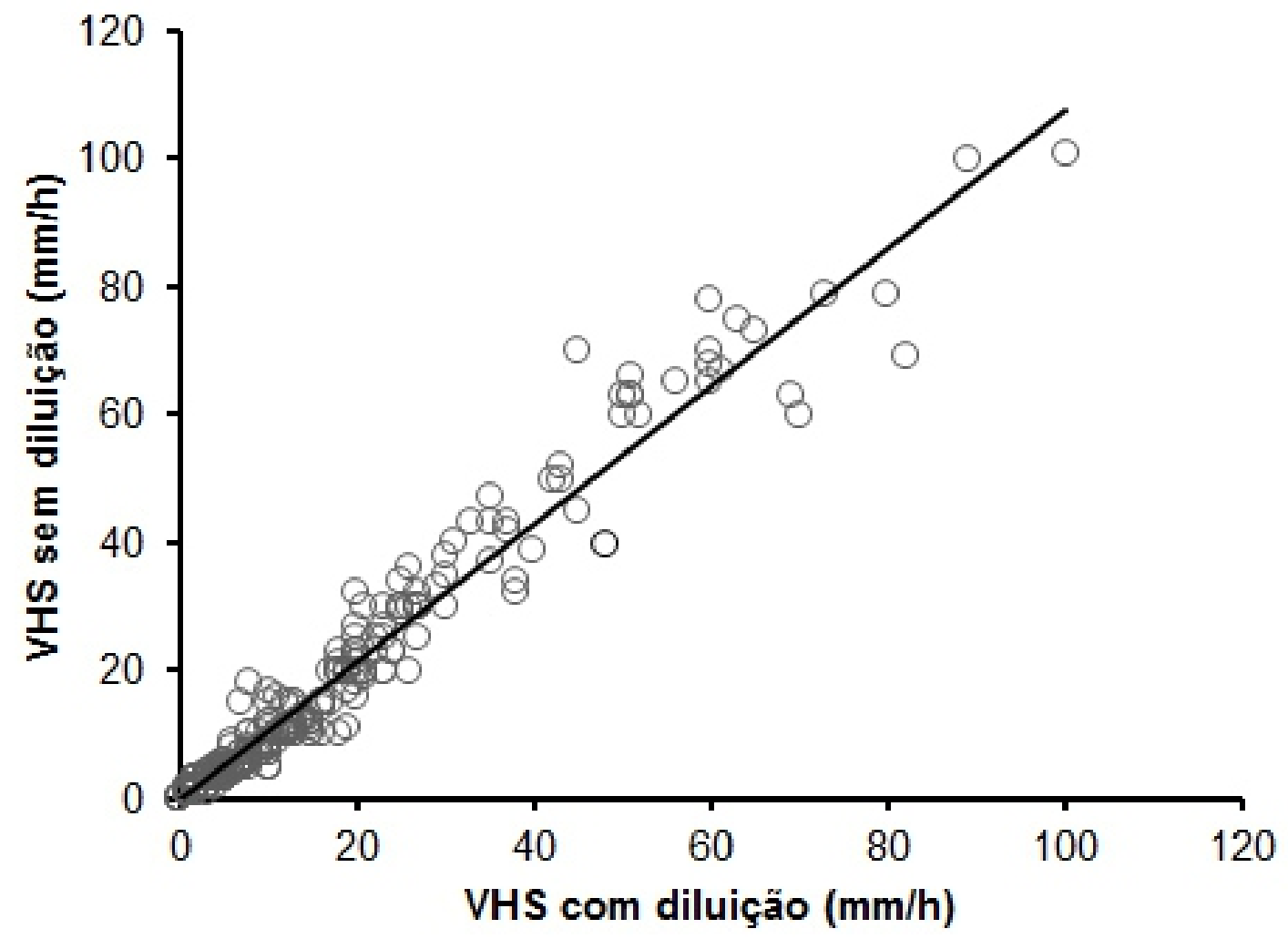

Regressão linear estimada $y=1,0742 x ; r=0,979 ; r^{2}-0,959$; tamanho amostral 326.

A análise estatística dos resultados pelo teste t para comparação entre duas médias revelou que não houve diferenças significantes entre o método de citrato e o VHS utilizando apenas o sangue anticoagulado com EDTA. Para uma melhor avaliação do desempenho dos métodos, os valores de VHS foram distribuídos em dois grupos: grupo 1 (valores de 0 a $30 \mathrm{~mm}$ ) e grupo 2 (valores acima de $30 \mathrm{~mm}$ ), sendo que a avaliação da correlação do grupo 2 está demonstrada no gráficos 2. Em VHS de valores acima de $30 \mathrm{~mm}$, esta correlação é menor $(r=0,87)$, sendo observado que, na maioria dos pacientes, os valores de VHS são maiores no método com EDTA quando comparados com o método que utiliza o citrato, com um erro sistemático de $9 \%$. 
FIGURA 2 - Correlação entre os dois métodos de determinação do VHS para valores acima de $30 \mathrm{~mm} / \mathrm{h}$

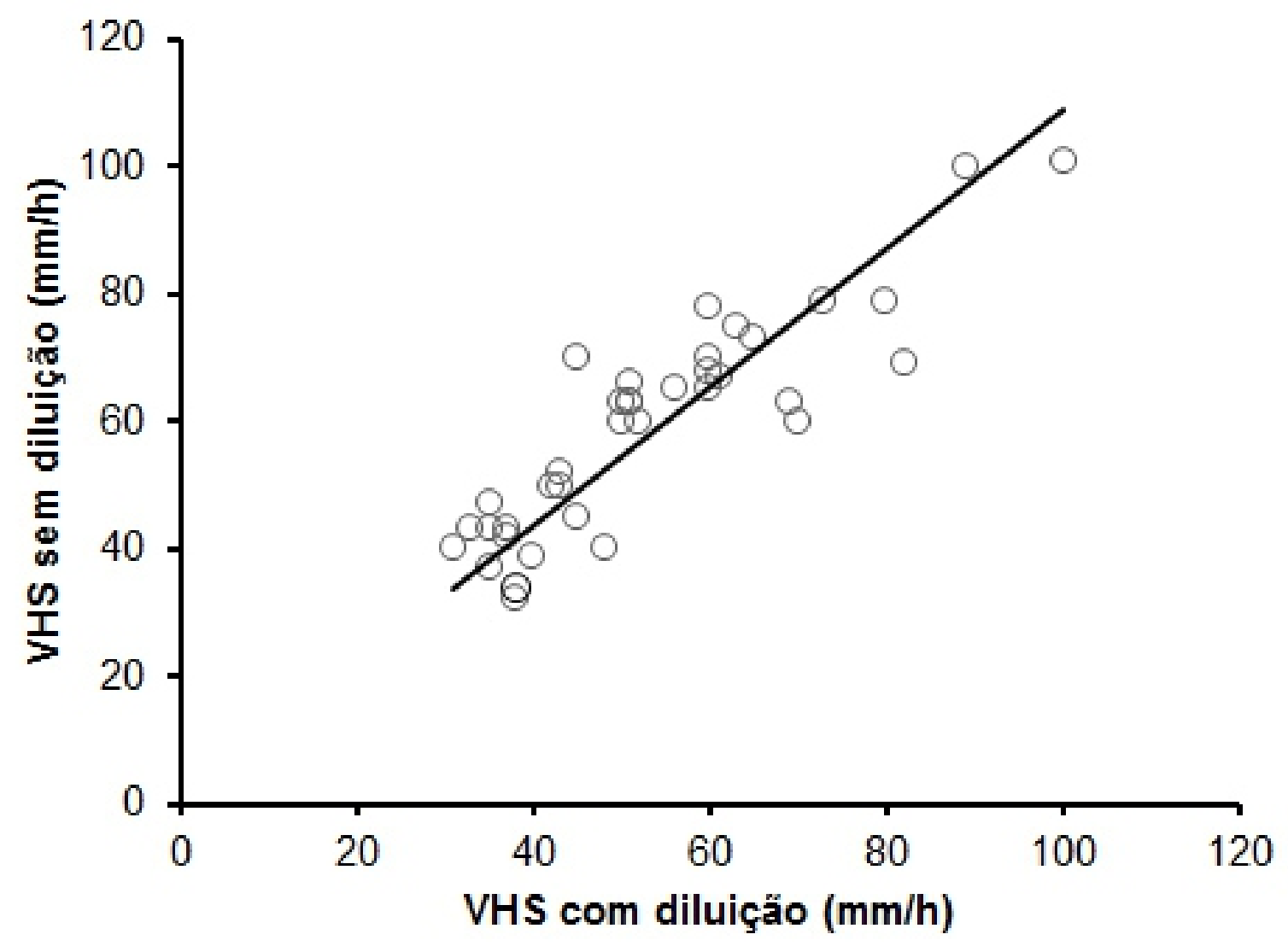

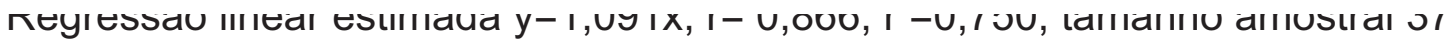

\section{DISCUSSÃO}

Apesar de muitas controvérsias sobre o valor real da medida da VHS, não há duvida de que esse exame constitui um importante parâmetro auxiliar para detecção de alterações na composição do plasma devido a diversas patologias ou condições que favoreçam o fenômeno de separação dos eritrócitos do plasma (DUSSE,2002). Existem, atualmente, vários métodos laboratoriais para verificar a existência de marcadores de resposta inflamatória. A velocidade de hemossedimentação é um teste simples e de baixo custo que tem sido usado, há mais de um século com este objetivo (COLLARES, 2004). O International Committee for Stardardization in Haematology, ICSH, 1977, considera aceitável algumas modificações introduzidas no método originalmente proposto por Westergren, entre elas a substituição do tubo de vidro por tubo plástico e o uso de EDTA como anticoagulante, desde que seja feita uma diluição do sangue, imediatamente antes de iniciar o exame, com uma parte de solução salina a $0,9 \mathrm{~g} / \mathrm{L}$ para quatro partes de sangue (DUSSE, 2002). O presente estudo foi motivado devido a grande utilização pela maioria dos laboratórios clínicos do método da VHS com EDTA, porém sem a sua prévia diluição com salina ou citrato. 
Neste trabalho não foi estipulado nenhum fator de inclusão ou exclusão específico, sendo que todas as amostras utilizadas proviam de uma rotina de um laboratório clínico de média complexidade. Os resultados deste estudo demonstraram que na média geral, a não-diluição do sangue com EDTA (método alternativo) não demonstrou nenhuma diferença significativa com relação ao método padrão ouro preconizado (gráfico 1).

Estratificando os valores de VHS acima de $30 \mathrm{~mm}$, observou-se um aumento significativo dos valores pelo método alternativo, ou seja, os valores da VHS por este método tendem a ser maiores do que os obtidos pela técnica com citrato. Como não foi instituído nenhum critério especifico de inclusão das amostras no trabalho, vários fatores podem contribuir para esta tendência. Os micrócitos sedimentam mais lentamente que os macrócitos, portanto, em casos de anemia, os valores da VHS podem variar, o que pode influenciar os resultados.

Toda a alteração da sedimentação parece estar correlacionada ao tipo de anticoagulante utilizado. Estudos demonstram que o citrato, nas concentrações empregadas pelo método de Westergren, provoca um retardamento na velocidade de sedimentação (DICKINSON, 1998).

Os resultados deste estudo corroboram com a teoria de que o citrato, pode retardar a sedimentação eritrocitária, principalmente em valores acima de $30 \mathrm{~mm}$. Por outro lado, a tendência do método alternativo de apresentar valores de VHS maiores do que o método com citrato pode ser explicada pela maior proximidade dos eritrócitos sem o uso de um diluente, o que aumenta a aproximação das células para a formação do rouleaux, sendo que esta formação é o mecanismo principal para a sedimentação dos eritrócitos.

Como no método com citrato existe uma diluição de $20 \%$, fazendo com que os eritrócitos estejam mais dispersos no plasma o que aumenta o tempo de formação do rouleuax. No método alternativo, provavelmente este fenômeno é menor, o que explicaria os resultados obtidos. Outro aspecto importante é de que a correlação entre os métodos foi menor quando os valores da VHS foram separados em acima e abaixo de $30 \mathrm{~mm}$. Este valor foi escolhido, por ser na maioria das vezes, considerado o limite de normalidade, desta forma, o método alternativo por apresentar valores maiores pode indicar processos inflamatórios onde o mesmo não existe (resultados falso-positivos).

Conclui-se com este trabalho que a utilização ou não de um diluente possui influência sobre os resultados da VHS, principalmente em valores acima dos limites de referência, sendo este um fator limitante na sensibilidade do método escolhido. Os resultados obtidos demonstram que o método com citrato tende a ter maior sensibilidade em demonstrar processos inflamatórios, visto que possui menor influência no potencial zeta, o que indicaria, melhor performance de diagnóstico e controle de doenças inflamatórias crônicas. 


\section{REFERÊNCIAS}

BEDELL S.E.; BUSH B.T. Erythrocyte sedimentation rate: from folklore to facts. Am J Med 1985; 78:10001-7.

COLLARES G.B.; VIDIGAL P.G. Recomendações para o uso da velocidade de hemossedimentação. Rev Med Minas Gerais 2004;14(1):52-7.

DICKINSON B. Avaliação da utilização de dois anticoagulantes e tempos diferentes para a realização do VHS, método Westergren. Sistema Vacutainer, 1998.

DUSSE L.M.; RIBEIRO A.P.M.; LAGES G.F.G.; GODOI L.C.; SOARES A. L.; VIEIRA L.M.; CARVALHO M. G. Avaliação da velocidade de hemossedimentação (VHS) utilizando o método de Westergren (original e modificado) e os sistemas Vacuette e Sedivette. Rev Bras Anal Clin 2002;34(2):103-105.

International Council for Standardization in Haematology (Expert Panel on Blood Rheology): ICSH recommendations for measurement of erythrocyte sedimentation rate. J Clin Pathol 1993;46:198-203.

LANZARA G.A.; PROVENZA J.R.; BONFIGLIOLI R.: Velocidade de hemossedimentação (VHS) de segunda hora: qual o seu valor? Rev Bras Reumatol, $2001 ;(4): 41-7 / 8$

SOX H.C. JR; LIANG M.H. The erythrocyte sedimentation rate: guidelines for rational use. Ann Intern Med, 1986;104:515-23. 